\title{
THE LONG-TERM RESULTS OF UNREPAIRED TEARS OF THE POSTERIOR CRUCIATE LIGAMENT
}

\author{
D. J. DANDY, R. J. PUSEY \\ From Addenbrooke's Hospital, Cambridge and Newmarket General Hospital, Suffolk
}

\begin{abstract}
A group of 20 patients with ruptures of the posterior cruciate ligament treated either by early mobilisation or by plaster immobilisation is described. The functional results bore no relationship to the degree of laxity of the ligament and 18 of the 20 patients considered that their symptoms did not warrant the discomfort and inconvenience of reconstruction of the ligament. It is suggested that many patients with posterior cruciate insufficiency have a good functional result, and that the place of routine repair of such injuries should be questioned.
\end{abstract}

Reports of the morbidity from ruptures of the posterior cruciate ligament vary widely. Fairbank (1944) reported that serious instability was exceptional after rupture of either cruciate ligament of the knee and did not advise repair, whereas others (Abbott et al. 1944; Hughston et al. 1980) report that repair of the ligament is essential if a painful and unstable joint is to be avoided. Early operation and repair is justified only if it offers a better long-term result than conservative treatment, but since no reports of the long-term results of untreated posterior cruciate ruptures could be found in the literature, the indications for early repair cannot be defined with confidence. To help in the investigation of this problem a group of patients is described with poor long-term results from untreated ruptures of the posterior cruciate.

\section{MATERIAL AND METHODS}

Patients were considered to have a rupture of the posterior cruciate ligament if they presented with a history of major trauma to the knee preceding the onset of their symptoms, and had a posterior drawer sign. Patients were excluded if repair or reconstruction of a ligament had been performed, if radiographs showed that the tibial attachment of the ligament had been avulsed with a block of bone, or if evidence of damage to another major ligament of the knee apart from the posterior cruciate was present. Twenty patients, with a mean age of 31 years (range 18 to 60 ), satisfied these criteria; 17 were men. The mean time between injury and review was 7.2 years (range 1 to 38 ). All patients were referred for an orthopaedic opinion because of symptoms arising from their injured knee, and therefore represent only those patients with unsatisfactory results. It is not known how many patients became. free of symptoms after their injury, or how many with residual symptoms have not been referred for an orthopaedic opinion.

The most common type of injury was a blow to the uppermost part of the tibia with the knee flexed (10 patients), but hyperextension injuries (four patients) and injuries caused by indirect violence (six patients) also occurred. In three patients, the injury was recognised

and the knee immobilised in a plaster of Paris cast for six weeks with the knee extended. In the remainder, the injury was either undiagnosed or treated by early active mobilisation. One patient had subsequently undergone medial meniscectomy, and another a lateral meniscectomy; neither had any improvement in their symptoms. Assessment. A detailed clinical history was taken from all patients, and the principal symptoms recorded. The details of the operation for posterior cruciate reconstruction, including a period of cast immobilisation and rehabilitation, were also explained and the patients were asked if they considered that their symptoms warranted such a procedure. The criteria described by Hughston et al. (1980) were also used to classify the functional, subjective and objective results as good, fair or poor, and the degree of posterior cruciate laxity recorded with a goniometer designed specifically for this purpose. The goniometer, which compares the relative position of the front of the tibial tubercle and the patella of the injured knee with that of the other leg, is shown in Figures 1 and 2 . To use the goniometer, the patient was asked to lie supine, with the knees flexed to 90 degrees. The fronts of both knees were examined to ensure that the tibial tubercles were comparable in size because any asymmetry, such as that resulting from OsgoodSchlatter's disease, would invalidate the readings. After the goniometer (a) had been placed over the tibial crest of the uninjured limb with the pad (b) applied to the tibial tubercle, the arm (c) was moved so that the tip rested lightly on the patella. The scale (d) was then moved so that the needle (e) was at 0 degrees. The scale was held firmly and the goniometer applied to the injured limb in the same way. The arm (c) moves as it comes into contact with the patella and the extent of the posterior sag was recorded in degrees on the scale (d). The test was repeated three times and the mean of the three readings recorded. The patient was asked not to contract the hamstrings when the readings were taken; only slight variation in the readings occurs even if the hamstrings are contracted forcefully. Less than two degrees of sag was considered to correspond with laxity of $1+$ on Hughston's scale, two to five degrees with $2+$ and more than five degrees with $3+$.

In 10 patients the posterior cruciate ligament was examined arthroscopically either from the posteromedial approach (Fig. 3) or from the anterolateral approach using a 70-degree telescope passed through the intercondylar notch. Arthroscopy was only undertaken if the symptoms were considered bad enough to warrant reconstruction of the posterior cruciate, or if there was a possibility that there might also be a meniscal injury or some other internal derangement of the knee.

D. J. Dandy, FRCS, Consultant Orthopaedic Surgeon $\{$ Addenbrooke's Hospital, Hills Road, Cambridge CB2 2QQ, England R. J. Pusey, FRCS, Senior Orthopaedic Registrar $\}$ and Newmarket General Hospital, Newmarket, Suffolk, England. Requests for reprints should be sent to Mr D. J. Dandy, Kennels Farmhouse, Great Wilbraham, Cambridge CB1 5JW, England. (1) 1982 British Editorial Society of Bone and Joint Surgery 0301-620X/82/1017-0092 $\$ 2.00$ 


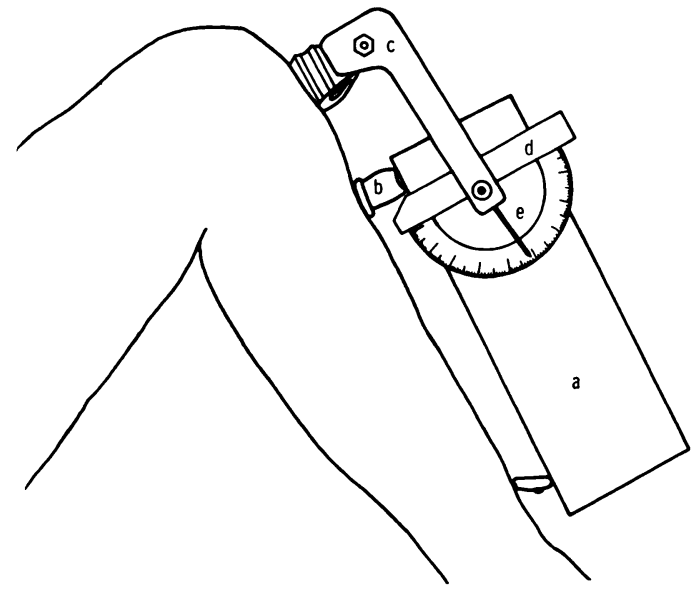

Fig. 1

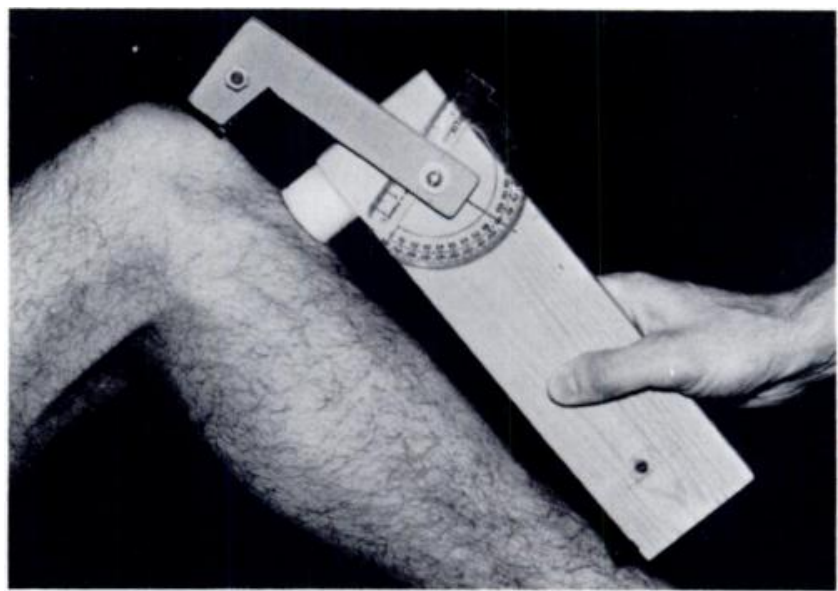

Fig. 2

Figures 1 and 2-The goniometer used to measure the degree of posterior cruciate laxity.

Since muscular activity tends to accentuate the physical signs of a posterior cruciate lesion, examination under anaesthetic was not performed. In this respect, injuries of the posterior cruciate ligament differ from lesions of the anterior cruciate and collateral ligaments of the knee.

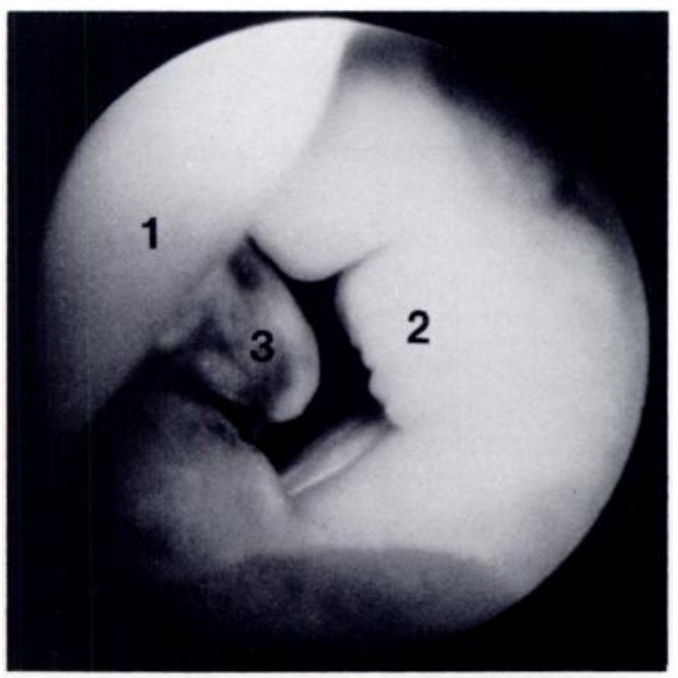

Fig. 3

The arthroscopic appearance of a ruptured posterior cruciate ligament showing the medial femoral condyle (1), the posterior capsule (2) and the posterior cruciate ligament stub (3) from the posteromedial approach.

\section{RESULTS}

The commonest symptoms were discomfort when weight was put on the semi-flexed knee, for example when descending stairs or a slope, starting to run, or picking up the handles of a loaded wheelbarrow. A feeling of unsteadiness and insecurity of the knee was also common. The symptoms reported by the 20 patients are set out in Table I. After explanation of the details of reconstruction of the posterior cruciate and the associated period of immobilisation and rehabilitation, only two of the 20 patients considered that their symptoms justified the economic problems and discomfort of the procedure.

Table II shows the subjective, objective and functional assessment of the knee made according to the criteria of Hughston et al. (1980). It was notable that there was no correlation between the functional and

Table I. Principal symptoms reported by the 20 patients

\begin{tabular}{|l|c|}
\hline \multicolumn{1}{|c|}{ Symptoms } & $\begin{array}{c}\text { Number of } \\
\text { patients }\end{array}$ \\
\hline Aches on walking long distances & 14 \\
Pain on walking down steps or stairs & 11 \\
Pain on squatting & 11 \\
Gives way while walking on uneven ground & 9 \\
Gives way on running straight & 4 \\
Gives way on going down stairs & 6 \\
Swells on walking & 4 \\
\hline
\end{tabular}

objective ratings, most patients having a good or fair functional result in the presence of a positive posterior sag sign, and other patients having severe symptoms despite little laxity of the ligament. No patient could be graded as good on the objective scale since this would require a negative posterior drawer sign while a positive sign is the minimal criterion for inclusion in this study.

Table II. Subjective, objective and functional grading for the 20 patients

\begin{tabular}{|l|c|c|c|}
\hline & Good & Fair & Poor \\
\hline Subjective & 8 & 10 & 2 \\
Objective & 0 & 8 & 12 \\
Functional & 5 & 11 & 4 \\
\hline
\end{tabular}


Table III. Sporting or recreational activities for 17 of the 20 patients

\begin{tabular}{|c|c|}
\hline & $\begin{array}{c}\text { Number of } \\
\text { patients }\end{array}$ \\
\hline Before accident & 17 \\
After accident: & 14 \\
Squash & 4 \\
Cricket & 3 \\
Football & 3 \\
Tennis & 1 \\
Rugby & 1 \\
Hiking & 1 \\
Badminton & 1 \\
\hline
\end{tabular}

Only three patients were unable to pursue sporting or recreational activities to their satisfaction because of their injury (Table III).

\section{DISCUSSION}

Neither this paper, nor that of Hughston et al. (1980), demonstated any correlation between the amount of ligamentous instability and the severity of the patient's symptoms. Some patients had an excellent functional result despite an obvious drawer sign, whereas others had disabling symptoms with only slight instability and this disparity is the essence of the conundrum of the posterior cruciate.

Rupture of the posterior cruciate ligament is not an uncommon injury in motorcyclists or motorists involved in rapid deceleration injuries but the lesion often escapes diagnosis at the time of injury. In this study, the lesion was only diagnosed immediately after injury in three of the 20 patients, which indicates that there are many patients with undiagnosed ruptures of the posterior cruciate ligament who have not found it necessary to seek the advice of an orthopaedic surgeon.

Examination showed no difference between those patients with severe symptoms, and those with mild symptoms. One possible reason for this difference might be the rupture of secondary supporting structures at the back of the knee, but this seems unlikely since Butler, Noyes and Grood (1980) have shown that the posterior cruciate ligament was responsible for 95 per cent of the posterior stability of the knee, which suggests that rupture of this ligament has an all or none effect.

If we accept that some patients remain free of symptoms despite the lack of a posterior cruciate ligament, the indications for the repair of acute posterior cruciate ruptures must be questioned. Hughston $e t$ al. (1980) reported the results of 29 acute repairs, of which all except three of the 20 examined had good results, yet 20 of these 29 patients had no evidence of posterior cruciate rupture on examination under anaesthetic; the lesions were discovered only when the ligament was explored surgically. Many surgeons agree with Trickey (1980) that the posterior drawer sign is always positive when the posterior cruciate ligament is completely torn. It can be argued that those patients with clinically intact ligaments could be treated equally well by conservative means, and that the exposure and dissection of such a ligament could jeopardise the chances of natural repair.

Trickey (1968) reported the results of reattachment of the tibial insertion of the posterior cruciate ligament in 17 patients, in 13 of whom the body of the ligament was intact but a block of bone had been avulsed. Such patients have been excluded from the present study. Torisu (1977) reported a series of 21 ruptures of the posterior cruciate and claimed a satisfactory result in all including 12 treated conservatively, eight of whom were graded as excellent.

Since the patients in this study were referred specifically for symptoms arising from the injured knee, they represent only the poor results and therefore cannot be used as a control group with which to compare the results of routine immediate repair of the posterior cruciate ligament. However, even in this group of poor results, only four had a poor functional grading by the criteria of Hughston et al. (1980) and only two of the 20 patients considered that their symptoms were bad enough to warrant a period of six weeks immobilisation in a cast and subsequent physiotherapy. Since the period of immobilisation and rehabilitation is the same after an early or a late reconstruction, the wisdom of routine early repair is open to question. The experience of Trickey (1968) supports immediate reattachment of the tibial insertion of the ligament when this has been avulsed, but the present study would suggest that for lesions of the body of the posterior cruciate ligament a more conservative approach can be justified.

We are grateful to Miss C. Tant for help in the preparation of this manuscript and to the Medical Photography and Illustration Department of Addenbrooke's Hospital, Cambridge, for the illustrations.

\section{REFERENCES}

Abbott LC, Saunders JB DeC M, Bost FC, Anderson CE. Injuries to the ligaments of the knee joint. J Bone Joint Surg 1944;26:503-21. Butler DL, Noyes FR, Grood ES. Ligamentous restraints to anterior-posterior drawer in the human knee. J Bone Joint Surg [Am] $1980 ; 62-A: 259-70$.

Fairbank HAT. Rehabilitation of the injured in this war and the last. Lancet 1944;2:131-4.

Hughston JC, Bowden JA, Andrews JR, Norwood LA. Acute tears of the posterior cruciate ligament. J Bone Joint Surg [Am] 1980;62-A:438-50.

Torisu T. Isolated avulsion fracture of the tibial attachment of the posterior cruciate ligament. J Bone Joint Surg [Am] 1977;59-A:68-72.

Trickey EL. Rupture of the posterior cruciate ligament of the knee.J Bone Joint Surg [Br] 1968;50-B:334-41.

Trickey EL. Injuries to the posterior cruciate ligament. Clin Orthop 1980;147:76-81. 